Теорія Ймовір. та Матем. Статист. Вип. 79, 2008
Theor. Probability and Math. Statist. No. 79, 2009, Pages 89-100 S 0094-9000(09)00783-2

Article electronically published on December 30, 2009

\title{
ASYMPTOTIC BEHAVIOR OF THE SOLUTION OF A LINEAR STOCHASTIC DIFFERENTIAL-DIFFERENCE EQUATION OF NEUTRAL TYPE
}

UDC 519.21

\author{
I. V. MALYK, E. F. TSAR'KOV, AND V. K. YASYNS'KY $\breve{I}$
}

\begin{abstract}
Necessary and sufficient conditions are found for the exponential mean square stability of a stochastic differential-difference linear equation of neutral type in the scalar case.
\end{abstract}

\section{INTRODUCTION}

The Lyapunov stability of solutions of deterministic differential-functional equations of neutral type is studied in many papers. A special place among these papers is occupied by Hale [15, Azbelev et al. [1, Kamenskir et al. [3, Khusainov and Shatyrko [13, 12, Slyusarchuk 9.

Kolmanovskiı and Nosov 8], Tsar'kov [14], and Khusainov [13] studied the behavior of solutions of stochastic differential-functional equations. The existence of a strong solution for a stochastic differential-functional equation of neutral type and generalizations of the second Lyapunov method are discussed by Kolmanovskiı̌ and Shaı̌khet in 2, Bereza and Yasyns'ky [ [5], and others.

In this paper, we obtain necessary and sufficient conditions for the exponential mean square stability of a stochastic differential-difference linear equation of neutral type and study the nonstable as well as the critical case.

\section{MAIN PART}

Let $(\Omega, F, \mathrm{P}, \Im)$ be a probability basis $[7]$ where $\Im \equiv\left\{F_{t}, t \geq 0\right\}$ is a filtration. Let a strong solution 2, 16] $x(t)=x(t, \omega) \in \mathbf{R}^{1}$ of the stochastic differential-difference linear equation of neutral type

$$
d\left\{D x_{t}\right\}=\left\{L x_{t}\right\} d t+\left\{G x_{t}\right\} d w(t)
$$

with the initial condition

$$
x_{0}=\varphi
$$

be defined on the probability basis $(\Omega, F, \mathrm{P}, \Im)$. Here

$$
x_{t} \equiv\{x(t+s),-h \leq s \leq 0\} \in C([-h, 0]),
$$

2000 Mathematics Subject Classification. Primary 60F15; Secondary 60G44.

Key words and phrases. Stochastic differential equations of neutral type, difference equations, eigenvalues. 
$\varphi \in C([-h, 0])$ is an $F_{0}$-measurable stochastic process, $w(t)=w(t, \omega)$ is a one-dimensional Wiener process adapted to $\Im=\left\{F_{t}, t \geq 0\right\}$, and where $D, L$, and $G$ are difference operators defined for $\psi \in C([-h, 0])$ by the following relations:

$$
\begin{array}{cl}
D \psi \equiv \psi(0)+\sum_{k=1}^{n} \delta_{k} \psi\left(-\tau_{k}\right), & 0<\tau_{1}<\tau_{2}<\cdots<\tau_{n} \leq h, \\
L \psi \equiv \alpha \psi(0)+\sum_{k=1}^{m} b_{k} \psi\left(-\lambda_{k}\right), & 0<\lambda_{1}<\lambda_{2}<\cdots<\lambda_{m} \leq h, \\
G \psi \equiv f \psi(0)+\sum_{k=1}^{q} g_{k} \psi\left(-\theta_{k}\right), & 0<\theta_{1}<\theta_{2}<\cdots<\theta_{q} \leq h .
\end{array}
$$

For the stochastic differential-difference linear equation of neutral type (1), (2), the existence and uniqueness (up to stochastic equivalence) hold in the case of a strong solution $x(t) \in \mathbf{R}^{1}$ such that $\mathrm{E}\left\{x^{2}(t)\right\}<\infty$ (see [5]). Along with equation (1) consider the deterministic differential-difference equation of neutral type [1, 15]

$$
d\left\{D y_{t}\right\}=\left\{L y_{t}\right\} d t
$$

with the initial condition

$$
y(t)=\varphi(t), \quad-h \leq t \leq 0 .
$$

First we mention some auxiliary results.

Lemma 2.1. If

$$
\sum_{k=1}^{n}\left|\delta_{k}\right|<1
$$

then the solution $y(t) \equiv 0$ of the deterministic differential-difference equation of neutral type (4), (5) is exponentially stable if and only if the roots of the characteristic quasipolynomial

$$
V(z) \equiv z\left(1+\sum_{k=1}^{n} e^{-z \tau_{k}} \delta_{k}\right)-a-\sum_{l=1}^{m} e^{-z \lambda_{l}} b_{l}
$$

belong to the left semi-plane of the complex plane $\mathbf{C}$, that is,

$$
\exists \rho>0, \quad \forall z \in \mathbf{C}: \quad V(z)=0 \Rightarrow \operatorname{Re} z<-\rho .
$$

Consider the Cauchy function $X(t)$ [15] as a solution of equation (4) satisfying the initial condition

$$
X(t) \equiv \mathbf{1}(t)= \begin{cases}0, & -h \leq t<0 \\ 1, & t=0\end{cases}
$$

Then the Cauchy function $X(t)$ can be represented in terms of the characteristic quasi-polynomial [4, 15].

Lemma 2.2. The Cauchy function $X(t)$ is of the form

$$
X(t)=\frac{1}{2 \pi i} \int_{\operatorname{Re} z=\mu} e^{z t} V^{-1}(z) d z
$$

where $\mu>-\rho$.

The following assertion holds. 
Theorem 2.1. A solution of the stochastic differential-difference linear equation of neutral type (1), (2) satisfies the stochastic integral equation

$$
x(t)=y(t)+\int_{0}^{t} X(t-s) G x_{s} d w(s),
$$

where $y(t)$ is a solution of (4), (2).

Proof. Since the neutral difference operator $D$ is linear, we get

$$
D x_{t}=D y_{t}+\int_{0}^{t} D X_{t-s} G x_{s} d w(s) \text {. }
$$

Substitute (12) into equation (1) and take into account the linearity of the difference operator $L$. Then we obtain

$$
\begin{aligned}
d D x_{t} & =d D y_{t}+d \int_{0}^{t} D X_{t-s} G x_{s} d w(s) \\
& =L y_{t} d t+D X_{0} G x_{t} d w(t)+\int_{0}^{t} d_{t} D X_{t-s} G x_{s} d w(s) \\
& =L y_{t} d t+G x_{t} d w(t)+\int_{0}^{t}\left(L X_{t-s} d t\right) G x_{s} d w(s) \\
& =L y_{t} d t+G x_{t} d w(t)+L\left\{\int_{0}^{t} X(t-s) d t G x_{s} d w(s)\right\} d t \\
& =L\left\{y_{t}+\int_{0}^{t} X(t-s) d t G x_{s} d w(s)\right\} d t+G x_{t} d w(t)=L x_{t} d t+G x_{t} d w(t) .
\end{aligned}
$$

The actions of the difference operator $L$ and the integration can be interchanged indeed, since $L$ is linear. Thus the stochastic process defined by (11) satisfies a stochastic differential-difference linear equation of neutral type. Theorem 2.1 is proved.

Now we define the notion of exponential mean square stability of a solution of a stochastic differential-difference linear equation of neutral type (1), (2).

Definition 2.1. The trivial solution $x(t) \equiv 0$ of the problem (1), (2) is called exponentially mean square stable if there are constants $M>0$ and $c>0$ such that

$$
\mathrm{E}|x(t)|^{2} \leq M e^{-c t} \mathrm{E}\|\varphi\|^{2}
$$

for all $t \geq 0$ and $\varphi \in C([-h, 0])$, where $\|\varphi\| \equiv \max _{-h \leq t \leq 0}|\varphi(t)|$ and $\mathrm{E}\{\cdot\}$ means the mathematical expectation.

We need the following notation and related results to find necessary and sufficient conditions for the exponential stability of the trivial solution $x(t) \equiv 0$ of a stochastic differential-difference linear equation of neutral type (1), (2).

Using the integral representation (11) of the strong solution of equation (1), and the representation (10) of the Cauchy function $X(t)$, we write an integral Volterra equation of neutral type for the stochastic process $\gamma(t) \equiv G x_{t}$ :

$$
\gamma(t) \equiv G y_{t}+\int_{0}^{t} H(t-s) \gamma(t) d w(s)
$$

where

$$
H(t) \equiv G X_{t}=\frac{1}{2 \pi i} \int_{\operatorname{Re} z=\mu} e^{z t} G_{1}(z) V^{-1}(z) d z
$$


and

$$
G_{1}(z) \equiv f+\sum_{i=1}^{q} g_{i} e^{-z \theta_{i}}
$$

To derive the latter equation, we apply the difference operator $G$ to both sides of equation (10) and obtain

$$
\begin{aligned}
G X_{t} & =G\left\{\frac{1}{2 \pi i} \int_{\operatorname{Re} z=\mu} e^{z t} V^{-1}(z) d z\right\}=\frac{1}{2 \pi i} \int_{\operatorname{Re} z=\mu}\left\{f e^{z t}+\sum_{k=1}^{q} g_{k} e^{z\left(t-\theta_{k}\right)}\right\} V^{-1}(z) d z \\
& =\frac{1}{2 \pi i} \int_{\operatorname{Re} z=\mu}\left\{f+\sum_{k=1}^{q} g_{k} e^{-z \theta_{k}}\right\} e^{z t} V^{-1}(z) d z=\frac{1}{2 \pi i} \int_{\operatorname{Re} z=\mu} G_{1}(z) e^{z t} V^{-1}(z) d z
\end{aligned}
$$

whence (14) follows.

Introduce the following notation:

$$
\begin{gathered}
M(t, \varphi) \equiv \mathrm{E}\left\{|x(t)|^{2} / F^{0}\right\}=\mathrm{E}\left\{|x(t)|^{2}\right\} \\
\Gamma(t, \varphi) \equiv \mathrm{E}\left\{|\gamma(t)|^{2} / F^{0}\right\}=\mathrm{E}\left\{|\gamma(t)|^{2}\right\}
\end{gathered}
$$

The conditional mathematical expectations above are equal to the corresponding unconditional mathematical expectations, since the stochastic processes $x(t)$ and $\gamma(t)$ are independent of the initial $\sigma$-algebra $F_{0}[7$.

Further we square the integral representation (11) of a strong solution of the stochastic differential-difference linear equation of neutral type and evaluate the mathematical expectations to obtain the following equation for the second moment $M(t, \varphi)$ :

$$
M(t, \varphi)=|y(t)|^{2}+\int_{0}^{t}|X(t-s)|^{2} \Gamma(s, \varphi) d s .
$$

In deriving this equality we take into account that the mathematical expectation of a Wiener-Itô integral is zero and that the mathematical expectation of the square of a Wiener-Itô integral is equal to the usual Riemann integral 7 .

Analogously we get

$$
\Gamma(t, \varphi)=\left|G y_{t}\right|^{2}+\int_{0}^{t}|H(t-s)|^{2} \Gamma(s, \varphi) d s .
$$

Next we prove the following auxiliary result.

Theorem 2.2. Let conditions (6) and (8) hold. Then a solution of the integral equation (18) is such that

$$
\exists C_{1}>0, \quad \exists C_{2}>0, \quad \forall \varphi \in C([-h, 0]): \quad \int_{0}^{\infty} \Gamma(t, \varphi) e^{t C_{2}} d t<C_{1}\|\varphi\|^{2}
$$

if and only if

$$
B \equiv \int_{0}^{\infty}|H(t)|^{2} d t<1 .
$$

Proof. Conditions (6) and (8) imply that there exists a constant $M>0$ such that

$$
|H(t)| \leq M e^{-\rho t}, \quad\left|G y_{t}\right| \leq M e^{-\rho t}\|\varphi\|
$$

for $\varphi \in C([-h, 0])$. This means that one can apply the Laplace transform to (18) and obtain the following equation:

$$
\int_{0}^{\infty} \Gamma(t, \varphi) e^{-z t} d t=\int_{0}^{\infty}\left|G y_{t}\right|^{2} e^{-z t} d t+\int_{0}^{\infty}|H(t)|^{2} e^{-z t} d t \int_{0}^{\infty} \Gamma(t, \varphi) e^{-z t} d t
$$


which is equivalent to the equation

$$
\int_{0}^{\infty} \Gamma(t, \varphi) e^{-\lambda t} d t=\int_{0}^{\infty}\left|G y_{t}\right|^{2} e^{-z t} d t\left(1-\int_{0}^{\infty}|H(t)|^{2} e^{-\lambda t} d t\right)^{-1}
$$

if $\operatorname{Re} z$ is sufficiently large. Without loss of generality we assume that $\operatorname{Im} z=0$. Note that the function $R(z)=\int_{0}^{\infty}|H(t)|^{2} e^{-z t} d s$, as a function of $z \in \mathbf{R}$, is continuous and that $R(z)$ is a decreasing function in the interval $(-\rho, \infty)$ if $\operatorname{Im} z=0$.

The sufficiency. Let relation (20) hold. Then there exists a number $\varepsilon>0$ such that

$$
\int_{0}^{\infty}|H(t)|^{2} e^{-z t} d t<1
$$

for $z \in[-\varepsilon, \infty)$. Then

$$
\begin{aligned}
\int_{0}^{\infty} \Gamma(t, \varphi) e^{-z t} d t & <\int_{0}^{\infty}\left|G y_{t}\right|^{2} e^{-z t} d t\left(1-\int_{0}^{\infty}|H(t)|^{2} e^{\varepsilon} d t\right)^{-1} \\
& \leq \int_{0}^{\infty}\left|G y_{t}\right|^{2} e^{\varepsilon} d t\left(1-\int_{0}^{\infty}|H(t)|^{2} e^{\varepsilon} d t\right)^{-1} \\
& \leq M^{2}\|\varphi\|^{2}(\rho-\varepsilon)^{-1}\left(1-\int_{0}^{\infty}|H(t)|^{2} e^{\varepsilon} d t\right)^{-1}
\end{aligned}
$$

for $z \in\left[-\varepsilon, \infty\right.$ ). Inequality (24) implies condition (19) if we let $C_{2}=\varepsilon$ and

$$
C_{1}=M^{2}(\rho-\varepsilon)^{-1}\left(1-\int_{0}^{\infty}|H(t)|^{2} e^{\varepsilon} d t\right)^{-1} .
$$

The necessity. Let condition (19) hold and $\operatorname{Im} z=0$. We prove (20) by contradiction. Assume that (20) does not hold, that is,

$$
\int_{0}^{\infty}|H(t)|^{2} d t \geq 1
$$

Then $\int_{0}^{\infty}|H(t)|^{2} e^{-z t} d t>1$ for $z<0$. This means that

$$
\int_{0}^{\infty} \Gamma(t, \varphi) e^{-z t} d t=\int_{0}^{\infty}\left|G y_{t}\right|^{2} e^{-z t} d t\left(1-\int_{0}^{\infty}|H(t)|^{2} e^{-z t} d t\right)^{-1}<0,
$$

whence $\Gamma(t, \varphi)<0$ for $\operatorname{Re} z<0$. This is a contradiction, since $\int_{0}^{\infty} \Gamma(t, \varphi) e^{-\lambda t} d t>0$ by definition. Theorem 2.2 is proved.

Lemma 2.3. The integral on the left hand side of condition (20) is equal to

$$
B \equiv \int_{0}^{\infty}|H(t)|^{2} d t=\frac{1}{\pi} \int_{0}^{\infty}|G(i s)|^{2}|V(i s)|^{-2} d s .
$$

Proof. Using integral representation (15) in place of $H(t)$ we have

$$
H(t)=\frac{1}{2 \pi i} \int_{-\infty}^{\infty} G_{1}(i s) e^{z t} V^{-1}(i s) d s .
$$

Note that $G_{1}(i s) e^{z t} V^{-1}(i s)$ is the image of $H(t)$ under the Laplace transform, that is,

$$
G_{1}(i s) e^{z t} V^{-1}(i s)=\int_{0}^{\infty} e^{-i s t} H(t) d t .
$$


Then the Plancherel-Parseval equality [4] implies that

$$
\begin{aligned}
\int_{0}^{\infty}|H(t)|^{2} d t & =\frac{1}{2 \pi} \int_{-\infty}^{\infty}|G(i s)|^{2}|V(i s)|^{-2} d s \\
& =\frac{1}{2 \pi}\left(\int_{-\infty}^{0}|G(i s)|^{2}|V(i s)|^{-2} d s+\int_{0}^{\infty}|G(i s)|^{2}|V(i s)|^{-2} d s\right) \\
& =\frac{1}{2 \pi}\left(\int_{0}^{\infty}|G(-i s)|^{2}|V(-i s)|^{-2} d s+\int_{0}^{\infty}|G(i s)|^{2}|V(i s)|^{-2} d s\right) \\
& =\frac{1}{\pi} \int_{0}^{\infty}|G(i s)|^{2}|V(i s)|^{-2} d s .
\end{aligned}
$$

Lemma 2.3 is proved.

Theorem 2.3. Let conditions (6) and (8) hold for the coefficients of the stochastic differential-difference linear equation of neutral type and for roots of its characteristic quasi-polynomial (7). Then the condition

$$
B=\frac{1}{\pi} \int_{0}^{\infty}|G(i s)|^{2}|V(i s)|^{-2} d s<1
$$

is necessary and sufficient for the exponential mean square stability of a solution of the problem (1), (2), where $G(z) \equiv f+\sum_{r=1}^{q} g_{r} e^{-z \theta_{r}}$.

Further, if $B>1$, then in every neighborhood of zero there exists an initial function $\varphi(t)$ such that

$$
\lim _{t \rightarrow \infty} M\left\{|x(t)|^{2}\right\}=\infty .
$$

Proof. The sufficiency. Let condition (28) hold. Thus (20) holds, too. Conditions (6) and (8) imply that there exist constants $M_{2}>0$ and $c_{2}>0$ such that

$$
\begin{aligned}
\left|G y_{t}\right|^{2} e^{t c_{2}} \leq M_{2}\|\varphi\|^{2}, & |y(t)|^{2} e^{t c_{2}} \leq M_{2}\|\varphi\|^{2}, \\
|X(t)|^{2} e^{t c_{2}} \leq M_{2}, & |H(t)|^{2} e^{t c_{2}} \leq M_{2}
\end{aligned}
$$

for all $t>0$ and $\varphi \in C([-h, 0])$ (see [15]).

Note that the constant $c_{2}>0$ in the bounds (30) is the same as that in Theorem 2.2.

Inequalities (30) imply that

$$
\begin{aligned}
\Gamma(t, \varphi) e^{t c_{2}} & =\left|G y_{t}\right|^{2} e^{t c_{2}}+\int_{0}^{t}|H(t-s)|^{2} e^{(t-s) c_{2}} e^{s c_{2}} \Gamma(s, \varphi) d s \\
& \leq M_{2}\|\varphi\|^{2}+M_{2} \int_{0}^{t} e^{s c_{2}} \Gamma(s, \varphi) d s \leq M_{2}\left(1+c_{1}\right)\|\varphi\|^{2}
\end{aligned}
$$

for all $t>0$ and $\varphi \in C([-h, 0])$, where the constant $c_{1}$ is defined in the proof of Theorem 2.2.

Using inequalities (30) and (31) we obtain a bound for $M(t, \varphi)$ satisfying equation (17); namely,

$$
M(t, \varphi) \leq M_{2}\left(1+c_{1}\right)\|\varphi\|^{2} e^{-t c_{2}}
$$

for $t>0$ and $\varphi \in C([-h, 0])$.

This implies exponential stability (see condition (13) in Definition 2.1). The sufficiency is proved.

The necessity. Let conditions (6), (8) hold and let the solution $x(t) \equiv 0$ be exponentially stable, that is, $\mathrm{E}|x(t)|^{2} \leq M e^{-c t} \mathrm{E}\|\varphi\|^{2}$ for $t>0$ and $\varphi \in C([-h, 0])$, where $M>0$ 
and $c>0$. Then

$$
\begin{aligned}
\Gamma(t, \varphi) & =\mathrm{E}\left\{\left|G x_{t}\right|^{2}\right\} \leq(q+1)\left(|f|^{2}+\sum_{r=1}^{q}\left|g_{r}\right|^{2}\right) \max _{-h \leq \theta \leq 0} \mathrm{E}\left\{|x(t+\theta)|^{2}\right\} \\
& \leq M(1+q)\left(|f|^{2}+\sum_{r=1}^{q}\left|g_{r}\right|^{2}\right) e^{-c t}\|\varphi\|^{2} .
\end{aligned}
$$

This means that there exists a constant $A_{1}>0$ such that

$$
\int_{0}^{\infty} \Gamma(t, \varphi) e^{t c_{2} d t}<c_{1}\|\varphi\|
$$

Taking into account equality (26) of Lemma 2.3, we derive inequality (28) from Theorem 2.2 .

Further, equality (23) means that the poles of $1-\int_{0}^{\infty} \Gamma(t, \varphi) e^{-\lambda t} d t$ coincide with the zeros of the function

$$
1-\int_{0}^{\infty}|H(t)|^{2} e^{-\lambda t} d t
$$

and that $\int_{0}^{\infty}|H(t)|^{2} e^{-\lambda t} d t$ is a continuous decreasing function of a real argument $\lambda$ such that

$$
\lim _{\lambda \rightarrow \infty} \int_{0}^{\infty}|H(t)|^{2} e^{-\lambda t} d t=0 .
$$

Note that there exists $\lambda_{0}>0$ such that

$$
1-\int_{0}^{\infty}|H(t)|^{2} e^{-\lambda_{0} t} d t=0
$$

This means that the function $\int_{0}^{\infty} \Gamma(t, \varphi) e^{-\lambda t} d t$ has a pole at $\lambda=\lambda_{0}>0$. In its turn, the latter means that

$$
\lim _{t \rightarrow \infty} \Gamma(t, \varphi)=\lim _{t \rightarrow \infty} e^{\lambda_{0} t}=\infty,
$$

whence (29) follows. Theorem 2.3 is proved.

\section{The CRITICAL CASE}

Consider the behavior of a solution of the stochastic differential-difference linear equation of neutral type (1), (2) for $B=1$.

Theorem 3.1. Assume that

1) conditions (6), (8) hold;

2)

$$
B=\frac{1}{\pi} \int_{0}^{\infty}|G(i s)|^{2}|V(i s)|^{-2} d s=1
$$

3)

$$
f+\sum_{k=1}^{q} g_{k} \neq 0
$$

Then there exists an initial function $\varphi \in C[-h, 0]$ such that

$$
0<\lim _{t \rightarrow \infty} \mathrm{E}|x(t)|^{2}<\infty .
$$


Proof. Let a strong solution of equation (1) be constructed for the initial condition

$$
\varepsilon(t)= \begin{cases}0, & -h \leq t<0 \\ \varepsilon, & t=0\end{cases}
$$

Then $y(t)=\varepsilon X(t)$ is a solution of the deterministic equation (4), while equation (12) for $\Gamma(t, \varphi)$ becomes of the following form:

$$
\begin{aligned}
\int_{0}^{\infty} \Gamma(t, \varphi) e^{-z t} d t & =\varepsilon \int_{0}^{\infty}\left|G X_{t}\right|^{2} e^{-z t} d t+\int_{0}^{\infty}|H(t)|^{2} e^{-z t} d s \int_{0}^{\infty} \Gamma(t, \varphi) e^{-z t} d t \\
& =\int_{0}^{\infty}|H(t)|^{2} e^{-z t} d s\left(\varepsilon+\int_{0}^{\infty} \Gamma(t, \varphi) e^{-z t} d t\right)
\end{aligned}
$$

Hence

$$
\int_{0}^{\infty} \Gamma(t, \varphi) e^{-z t} d t=\frac{\varepsilon \int_{0}^{\infty}|H(t)|^{2} e^{-z t} d t}{\left(1-\int_{0}^{\infty}|H(t)|^{2} e^{-z t} d t\right)} .
$$

A relationship between the behavior of an original $\Gamma(t, \varphi)$ as $t \rightarrow \infty$ and that of its Laplace image as $z \rightarrow 0$ is well known, namely

$$
\lim _{t \rightarrow \infty} \Gamma(t, \varphi)=\varepsilon \lim _{z \rightarrow 0} \frac{z \int_{0}^{\infty}|H(t)|^{2} e^{-z t} d t}{\left(1-\int_{0}^{\infty}|H(t)|^{2} e^{-z t} d t\right)}
$$

see [6]. The limit as $z \rightarrow 0$ of the right hand side of equation (31) can be evaluated by using l'Hôpital's rule:

$$
\begin{aligned}
& \lim _{z \rightarrow 0} \frac{\varepsilon z \int_{0}^{\infty}|H(t)|^{2} e^{-z t} d s}{\left(1-\int_{0}^{\infty}|H(t)|^{2} e^{-z t} d t\right)}=\lim _{z \rightarrow 0} \frac{\left(\varepsilon z \int_{0}^{\infty}|H(t)|^{2} e^{-z t} d s\right)^{\prime}}{\left(1-\int_{0}^{\infty}|H(t)|^{2} e^{-z t} d t\right)^{\prime}} \\
& \quad=\varepsilon \lim _{z \rightarrow 0} \frac{\int_{0}^{\infty}|H(t)|^{2} e^{-z t} d s-z \int_{0}^{\infty}|H(t)|^{2} t e^{-z t} d s}{\int_{0}^{\infty}|H(t)|^{2} t e^{-z t} d t} \\
& \quad=\varepsilon \lim _{z \rightarrow 0} \frac{\int_{0}^{\infty}|H(t)|^{2} e^{-z t} d s}{\int_{0}^{\infty}|H(t)|^{2} t e^{-z t} d t}=\frac{\varepsilon}{\int_{0}^{\infty}|H(t)|^{2} t d t} .
\end{aligned}
$$

Conditions (8) imply that the integral in the denominator of the right hand side of (38) exists and

$$
\varepsilon=\varepsilon \int_{0}^{\infty}|H(t)|^{2} d t \leq \int_{0}^{\infty}|H(t)|^{2} t d t \leq K \int_{0}^{\infty}|H(t)|^{2} e^{\rho t / 2} d t=c<\infty
$$

where $K=\rho / 2$. Thus

$$
\frac{\varepsilon}{C} \leq \lim _{t \rightarrow \infty} \Gamma(t, \varphi) \leq 1
$$

Since $\Gamma(t, \varphi)=M\left\{G x_{t}\right\}^{2}$,

$$
\lim _{t \rightarrow \infty} \Gamma(t, \varphi)=\left(f+\sum_{k=1}^{q} g_{k}\right)^{2} \lim _{t \rightarrow \infty} M\left\{x^{2}(t)\right\}
$$

if the $\operatorname{limit}_{\lim } \rightarrow \infty \Gamma(t, \varphi)$ exists. Thus we derive from (40) and (41) that

$$
0<\frac{\varepsilon}{C}\left(f+\sum_{k=1}^{q} g_{k}\right)^{-2} \leq \lim _{t \rightarrow \infty} \mathrm{E}|x(t)|^{2} \leq\left(f+\sum_{k=1}^{q} g_{k}\right)^{-2}<\infty .
$$

Theorem 3.1 is proved. 
Along with equation (1) we consider the perturbed equation

$$
d\left\{D x_{t}\right\}=\left\{L x_{t}\right\} d t+(1+\alpha(t))\left\{G x_{t}\right\} d w(t) .
$$

Assume that condition (32) holds. We study the asymptotic stability and instability of the problem (42), (2) (in other words, of the problem (1), (2) under permanent perturbations).

By $\Re_{1}$, we denote the space of continuous in $[0, \infty)$ functions $\phi$ such that

$$
\exists \varepsilon \in(0,1), \quad \forall t>0: \quad(1+\phi(t))^{2}<1-\varepsilon .
$$

Let $\Re_{2}$ denote the space of continuous in $[0, \infty)$ functions $\phi$ such that

$$
\exists \varepsilon>0, \quad \forall t>0: \quad(1+\phi(t))^{2}>1+\varepsilon .
$$

Theorem 3.2. Let conditions (6), (8) hold and $B=1$. Then

A) the trivial solution of equation (42) is asymptotically stable in the mean square sense if $\alpha \in \Re_{1}$;

B) the trivial solution of equation (42) is unstable in the mean square sense if $\alpha \in \Re_{2}$.

Proof. We rewrite equation (42) in the form of an integral equation, namely

$$
x(t)=y(t)+\int_{0}^{t}(1+\alpha(s)) X(t-s) G x_{s} d w(s),
$$

where $y(t)$ is a solution of the problem (4), (2), while $X(t)$ is its fundamental solution, $t \geq 0$.

Indeed, applying the linear operator $D$ to (43) and then differentiating the result, we obtain

$$
\begin{aligned}
d D x_{t} & =d D y_{t}+d D\left\{\int_{0}^{t}(1+\alpha(s)) X(t-s) G x_{s} d w(s)\right\} \\
& =L y_{t} d t+D d\left\{\int_{0}^{t}(1+\alpha(s)) X(t-s) G x_{s} d w(s)\right\} \\
& =L y_{t} d t+\int_{0}^{t}(1+\alpha(s)) d D X(t-s) G x_{s} d w(s)+(1+\alpha(t)) G x_{t} d w(t) \\
& =L y_{t} d t+\int_{0}^{t}(1+\alpha(s)) L X(t-s) G x_{s} d w(s) d t+(1+\alpha(t)) G x_{t} d w(t) \\
& =L\left\{y_{t}+\int_{0}^{t}(1+\alpha(s)) X(t-s) G x_{s} d w(s)\right\} d t+(1+\alpha(t)) G x_{t} d w(t) \\
& =L x_{t}+(1+\alpha(t)) G x_{t} d w(t),
\end{aligned}
$$

whence (43) follows.

Using integral equation (43) and equality (18) we get

$$
\Gamma(t, \varphi)=\left|G y_{t}\right|^{2}+\int_{0}^{t}(1+\alpha(s))^{2}|H(t-s)|^{2} \Gamma(s, \varphi) d s .
$$

A) Let $\alpha \in \Re_{1}$. Then equality (44) implies that

$$
\Gamma(t, \varphi)<\left|G y_{t}\right|^{2}+(1-\varepsilon) \int_{0}^{t}|H(t-s)|^{2} \Gamma(s, \varphi) d s .
$$

Applying the Laplace transform to both sides of (45), we prove an inequality for images, namely

$$
\int_{0}^{\infty} \Gamma(t, \varphi) e^{-z t} d t<\int_{0}^{\infty}\left|G y_{t}\right|^{2} e^{-z t} d t+(1-\varepsilon) \int_{0}^{\infty}|H(t)|^{2} e^{-z t} d s \int_{0}^{\infty} \Gamma(t, \varphi) e^{-z t} d t
$$


Or

$$
\int_{0}^{\infty} \Gamma(t, \varphi) e^{-z t} d t<\int_{0}^{\infty}\left|G y_{t}\right|^{2} e^{-z t} d t\left(1-(1-\varepsilon) \int_{0}^{\infty}|H(t)|^{2} e^{-z t} d t\right)^{-1} .
$$

If $B=1$, then the pole of the right hand side of inequality (46) with the largest real part coincides with $\lambda_{0}$ for which

$$
\int_{0}^{\infty}|H(t)|^{2} e^{-\lambda_{0} t} d t=\frac{1}{1-\varepsilon}>1
$$

Since the Laplace transform with a real argument $(\lambda \in \mathbf{R})$ is a decreasing continuous function and $B=1$, we get $\lambda_{0}<0$. This means that $\Gamma(t, \varphi)$ behaves at $\infty$ like a function whose absolute value does not exceed $N e^{\lambda_{0} t}, N>0$, that is,

$$
\lim _{t \rightarrow \infty} \Gamma(t, \varphi)=0 \text {. }
$$

This proves A).

B) Let $\alpha \in \Re_{2}$. Then equality (44) implies that

$$
\Gamma(t, \varphi)>\left|G y_{t}\right|^{2}+(1+\varepsilon) \int_{0}^{t}|H(t-s)|^{2} \Gamma(s, \varphi) d s .
$$

Applying the Laplace transform to both sides of (49), we prove the following inequality for the images:

$$
\int_{0}^{\infty} \Gamma(t, \varphi) e^{-z t} d t>\int_{0}^{\infty}\left|G y_{t}\right|^{2} e^{-z t} d t+(1+\varepsilon) \int_{0}^{\infty}|H(t)|^{2} e^{-z t} d t \int_{0}^{\infty} \Gamma(t, \varphi) e^{-z t} d t
$$

or

$$
\int_{0}^{\infty} \Gamma(t, \varphi) e^{-\lambda t} d t>\int_{0}^{\infty}\left|G y_{t}\right|^{2} e^{-z t} d t\left(1-(1+\varepsilon) \int_{0}^{\infty}|H(t)|^{2} e^{-\lambda t} d t\right)^{-1} .
$$

If $B=1$, then the pole of the right hand side of (46) with the largest real part coincides with the number $\lambda=\lambda_{0}$ for which

$$
\int_{0}^{\infty}|H(t)|^{2} e^{-\lambda t} d s=\frac{1}{1+\varepsilon}<1 .
$$

Since the Laplace transform of a real argument $(\lambda \in R)$ is a decreasing function and $B=1$, we get $\lambda_{0}>0$. This means that $\Gamma(t, \varphi)$ behaves at $\infty$ like a function whose absolute value is not less than $\delta e^{\lambda_{0} t}$, that is,

$$
\lim _{t \rightarrow \infty} \Gamma(t, \varphi)=\infty
$$

whence B) follows. Theorem 3.2 is proved.

Remark 3.1 . The spaces $\Re_{1}$ and $\Re_{2}$ in Theorem 4.2 can be changed for "wider spaces", namely for

$$
\begin{aligned}
& \widetilde{\Re}_{1} \equiv\left\{\phi \in C^{0}([0, \infty]): \exists \varepsilon>0, \forall t \in[0, h]:(1+\phi(t))^{2}<1-\varepsilon ; \forall t>h:(1+\phi(t))^{2} \leq 1\right\}, \\
& \widetilde{\Re}_{2} \equiv\left\{\phi \in C^{0}([0, \infty]): \exists \varepsilon>0, \forall t \in[0, h]:(1+\phi(t))^{2}>1+\varepsilon ; \forall t>h:(1+\phi(t))^{2} \geq 1\right\}, \\
& \text { respectively. }
\end{aligned}
$$

Proof. We prove, for example, the part of Remark 3.1 related to the spaces $\Re_{1}$ and $\widetilde{\Re}_{1}$. Indeed, inequality (45) can be rewritten as follows:

$$
\Gamma(t, \varphi) \leq\left|G y_{t}\right|^{2}+(1-\varepsilon) \int_{0}^{t}|H(t-s)|^{2} \Gamma(s, \varphi) d s
$$


for $t \in[0, h]$ and

$$
\Gamma(t, \varphi) \leq\left|G y_{t}\right|^{2}+(1-\varepsilon) \int_{0}^{h}|H(t-s)|^{2} \Gamma(s, \varphi) d s+\int_{h}^{t}|H(t-s)|^{2} \Gamma(s, \varphi) d s
$$

for $t>h$.

Then inequality (46) becomes of the form

$$
\begin{aligned}
\int_{0}^{\infty} & \Gamma(t, \varphi) e^{-\lambda t} d t \\
& <\int_{0}^{\infty}\left|G y_{t}\right|^{2} e^{-z t} d t\left(1-\int_{0}^{\infty}|H(t)|^{2} e^{-\lambda t} d t+\varepsilon \int_{0}^{h}|H(t)|^{2} e^{-\lambda t} d t\right)^{-1} .
\end{aligned}
$$

Since $B=1$ and

$$
\int_{0}^{h}|H(t)|^{2} e^{-\lambda t} d t=K>0
$$

all poles of the right hand side of (53) belong to the left semi-plane of the complex plane. This means that $\Gamma(t, \varphi)$ behaves at $\infty$ like an exponential function with a negative index, that is, a solution of the perturbed equation (42) is exponentially stable in the mean square sense. Remark 3.1 is proved.

\section{BIBLIOGRAPHY}

1. N. V. Azbelev, V. P. Maksimov, and L. F. Rakhmatullina, Introduction to the Theory of Linear Functional-Differential Equations, Nauka, Moscow, 1991; English transl., World Federation Publishers Company, Atlanta, GA, 1995. MR1144998 (92j:34123); MR.1375462 (96m:34132)

2. E. A. Andreeva, V. B. Kolmanovskiı̌, and L. E. Shaĭkhet, Control of Systems with Aftereffect, Nauka, Moscow, 1992; English transl., V. B. Kolmanovskiı̌ and L. E. Shaı̆khet, American Mathematical Society, Providence, RI, 1996. MR 1185708 (93i:49001) MR.1415834

3. R. R. Akhmerov, M. I. Kamenskiı̌, A. S. Potapov, A. E. Rodkina, and B. N. Sadovskiı̌, The theory of equations of neutral type, Akad. Nauk SSSR, Vsesoyuz. Inst. Nauchn. i Tekhn. Informatsii, Mathematical Analysis 19 (1982), no. 1, 55-126, 232. (Russian) MR657948 (83j:34078)

4. R. Bellman and K. L. Cooke, Differential-Difference Equations, Academic Press, New YorkLondon, 1967. MR0147745 (26:5259)

5. Yu. V. Bereza and V. K. Yasyns'kyı̆, The existence of solutions of stochastic differential functional equations of the neutral type with Poissonian switchings, Visn. Kyiv. Univ., Ser. Fiz.-Mat. Nauky 5 (2002), no. 1, 19-27. (Ukrainian)

6. G. Doetsch, Introduction to the Theory and Application of the Laplace Transformation, Springer-Verlag, New York-Heidelberg, 1974; Translated from the second German edition. MR0344810(49:9549)

7. J. Jacod and A. N. Shiryaev, Limit Theorems for Stochastic Processes, Nauka, Moscow, 1994; English transl. of the second edition, Springer-Verlag, Berlin, 2003. MR 1943877 (2003j:60001)

8. V. B. Kolmanovskil and V. R. Nosov, Stability and Periodic Modes of Adaptable Systems with Aftereffect, Nauka, Moscow, 1981. (Russian) MR641554 (83g:34001)

9. V. Yu. Slyusarchuk, The Absolute Stability of Dynamical Systems with Aftereffect, UDUVGP, Rivne, 2003. (Ukrainian)

10. I. Ya. Spektorskiı̌, A generalization of the constant variation formula for a linear nonhomogeneous stochastic equation, Problemy Upravlen. Inform. 1 (1998), no. 5, 107-112, 158. (Russian) MR.1700664

11. R. Horn and C. Johnson, Matrix Analysis, Cambridge University Press, Cambridge, 1985. MR832183 (87e:15001)

12. D. Ya. Khusainov, Estimates for the stability of solutions of systems of functional-differential equations of neutral type, Ukrain. Mat. Zh. 1 (1991), no. 9, 1123-1135; English transl. in Ukrainian Math. J. 1 (1991), no. 9, 1053-1063. MR1149573 (92k:34102)

13. D. Ya. Khusainov and A. V. Shatyrko, The method of Lyapunov functions in the studies of the stability of differential-functional systems, Kiev University, Kiev, 1997. (Russian) MR1486825 (2000k:34124)

14. E. F. Tsar'kov, Random Perturbations of Differential-Functional Equations, Zinatne, Riga, 1989. (Russian) MR1036733 (90m:34164) 
15. J. Hale, Theory of Functional Differential Equations, Springer, Berlin, 1978. MR0508721 $(58: 22904)$

16. I. I. Gihman and A. V. Skorohod, Stochastic Differential Equations, Springer, Berlin, 1972. MR0346904 (49:11625)

Department of Mathematical and Applied Statistics, Faculty for Applied Mathematics, Chernivtsi Fed'Kovych National University, Kotsyubyns'kyi Street 2, Chernivtsi 58000, UKRAINE

E-mail address: M_Ihor_V@rambler.ru

Department of Probability Theory and Mathematical Statistics, Riga Technical UniverSity, Riga, Latvia

E-mail address: carkovs@livas.lv

Department of Mathematical and Applied Statistics, Faculty for Applied Mathematics, Chernivtsi Fed'kovych National University, Kotsyubyns'kyi Street 2, Chernivtsi 58000, UKRAINE

E-mail address: yasinsk@cv.ukrtel.net

Received 20/FEB/2008

Translated by N. SEMENOV 